\title{
A multiphase multiscale model for nutrient limited tissue growth, Part II: A simplified description
}

\author{
E.C. Holden ${ }^{1}$, S.J. Chapman ${ }^{2}$, B.S. Brook ${ }^{1}$ \& R.D. O'Dea ${ }^{1}$ \\ ${ }^{1}$ Centre for Mathematical Medicine and Biology, \\ School of Mathematical Sciences, \\ University of Nottingham, University Park, \\ Nottingham, NG7 2RD, UK \\ ${ }^{2}$ Mathematical Institute, University of Oxford, Radcliffe Observatory Quarter, \\ Woodstock Road, Oxford OX2 6GG, UK
}

July 8, 2019

\begin{abstract}
In this paper, we revisit and extend our recent work (Holden et al. (2018) A multiphase multiscale model for nutrient limited tissue growth, The ANZIAM Journal, 59(4), 499-532), that considers the derivation of an effective macroscale description suitable to describe the growth of biological tissue within a porous tissue-engineering scaffold. The underlying tissue dynamics is described as a multiphase mixture, thereby naturally accommodating features such as interstitial growth and active cell motion. Via a linearisation of the underlying multiphase model (whose nonlinearity poses significant challenge for such analyses), we obtain, by means of multiple-scales homogenisation, a simplified macroscale model that nevertheless retains explicit dependence on both the microscale scaffold structure and the tissue dynamics, via so-called unit cell problems that provide permeability tensors to parameterise the macroscale description. In our previous work, the cell problems retain macroscale dependence, posing significant challenges for computational implementation of the eventual macroscopic model; here, we obtain a decoupled system whereby the quasi-steady cell-problems may be solved separately from the macroscale description. Moreover, we indicate how the formulation is influenced by a set of alternative microscale boundary conditions.
\end{abstract}

\section{Introduction}

Tissue growth is a complex and inherently multiscale phenomenon, whose unified description requires the integration of insight obtained at one scale with observations at another. For example, growth processes (or disease manifestation) at the organ scale are driven by microscopic events at the (sub-)cellular scale that themselves are influenced by macroscopic dynamics. Such complexity leads inevitably to formulations that are analytically and computationally intractable, or are otherwise highly idealised. For this reason, a significant area of research is dedicated to developing various mathematical and computational techniques that enable efficient coupling between dynamics occurring on multiple scales (see, e.g. $[1,14,21]$ and references therein).

This article follows on from Holden et al. [9], in which the derivation of a coarse-scale description of the tissue dynamics that nevertheless retains aspects of tissue microstructure and cell behaviour is considered. Here, we show that the formulation derived therein may be significantly simplified. To effect this, we again employ the method of multiple-scale asymptotic homogenisation (see, e.g. $[2,12]$, and $[7,22]$ for reviews). The key feature of this approach is to derive suitable macroscale equations from an underlying microscale description, rather than stating them ab initio. Coupling to the 
microscale physics is enabled by defining suitable problems on a prototypical 'unit cell', by which to specify effective coefficients in the macroscale description.

A series of recent studies have employed these methods in a biological setting to consider growing tissues. We refer the interested reader to Holden et al. [9] for more extended motivation and background literature, but note here that a common feature of such studies is the highly idealised representation of growth. This deficiency was addressed in Holden et al. [9], wherein we instead employed a multiphase model to describe the microscale tissue dynamics. In particular, this approach accommodates nutrient-limited interstitial growth, as well as cell aggregation and repulsion. An effective macroscale description is obtained via a linearisation that ameliorates problems associated with complex mass-transfer considered in the multiphase model (see [4] for a discussion), and allows one to obtain via homogenisation techniques a more tractable description that permits coupling between micro- and macro-scale processes. The derived model comprises macroscopic PDEs describing the evolution of the cell population, nutrient concentration, and cell and interstitial fluid flow. These are coupled to the underlying microscale structure and dynamics via suitable cell problems. Importantly, and in contrast to other similar studies, these unit cell problems are themselves parameterised by the macroscale dynamics, so that the micro- and macro- scale descriptions are fully coupled. Here, we modify the analysis of [9] to obtain the more standard structure, obtaining a system in which the quasi-steady cell-problems may be solved separately from the macroscale description, thereby greatly simplifying the computational difficulty that would be associated with fully-coupled multiscale descriptions. We reiterate that the effective description that we obtain embeds microscale modelling choices, both in terms of the model governing equations and the associated boundary conditions describing, e.g., cell-scaffold interactions or nutrient transport into the growing tissue. We therefore consider several alternative microscopic boundary conditions to indicate how the macroscopic formulation is modified.

This paper is organised as follows. In Section 2 we briefly recapitulate the microscale model and boundary conditions of [9]. In Section 3 we perform a multiscale homogenisation to obtain an effective macroscale description, and consider alternative microscopic boundary conditions in Section 4; Section 5 summarises our results and provides suggestions for future work.

\section{Model formulation}

We consider a model of broad relevance to tissue engineering applications where tissue growth occurs on a structured periodic scaffold, such as can be achieved through the use of 3D printing [11, 25]. We emphasise that this article follows on from Holden et al. [9], and the underlying model set-up considered herein is identical to that study (itself following closely [13], that builds on the general theory of multiphase porous flow developed in $[3,8,15]$ ), and so only a very brief descriptive summary is included here.

The microscale domain is denoted $\Omega$, with boundary $\partial \Omega$, and has characteristic lengthscale $l^{*}$. This domain comprises scaffold, $\Omega_{S}$, tissue $\Omega_{T}$ and interstitial fluid $\Omega_{I}$. The scaffold boundary is denoted by $\partial \Omega_{S}$ and the tissue-interstitial boundary by $\Gamma$; see Figure 1 for a schematic diagram. The macroscopic lengthscale (associated with the full extent of the scaffold) is denoted $L$. The lengthscales in question are well-separated such that

$$
\varepsilon=\frac{l}{L} \ll 1 .
$$

We model the porous scaffold material as a rigid solid, and the tissue as a two phase mixture of cells (with volume fraction $\theta_{n}$ ) and water $\left(\theta_{w}\right)$ which covers the scaffold, whilst the interstitial space contains only water. Both cells and water are modelled as viscous fluids (with pressure $p_{i}$ and velocity $\boldsymbol{v}_{i} ; i \in\{n, w, I\}$ ), described by a Stokes flow. Increase in the cell volume fraction of the mixture depends on the concentration of a generic diffusible nutrient (denoted $c_{i} ; i \in\{T, I\}$ ), as well as the availability of water. Tissue growth is represented by movement of the boundary $\Gamma$, occuring as a consequence of nutrient-limited phase transition within the tissue domain or cell aggregation/repulsion (these being effected by the specification of suitable additional pressures $\phi_{n}\left(\theta_{n}\right)$ in 


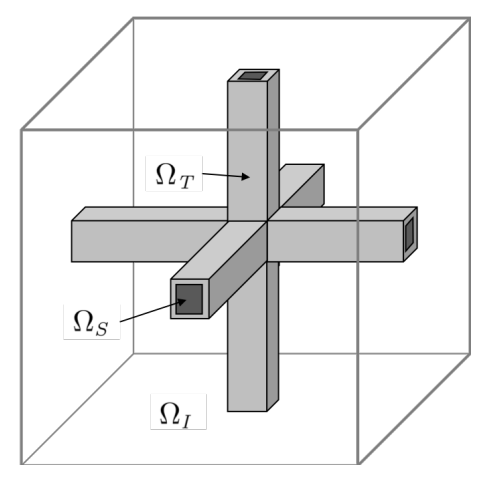

Figure 1: Schematic diagram of the microscale domain $\Omega$ illustrating a periodic scaffold covered with a layer of tissue, indicating the scaffold, $\Omega_{S}$, tissue $\Omega_{T}$ and interstitial fluid $\Omega_{I}$ domains. The scaffold boundary is denoted by $\partial \Omega_{S}$ and tissue-interstitial boundary by $\Gamma$.

the cell phase). Coupling between the tissue domain and the surrounding interstitium is described by suitable mass flux conditions across the tissue-interstitium interface, as well as continuity of tangential velocity, stress and nutrient concentration. No-slip and no-penetration is imposed on the scaffold surface. We emphasise that our model describes a complex free-boundary problem in which the interface position $\Gamma$ is not known, and should be determined as part of the solution. However, in the multiscale analysis that follows, the boundary velocity remains undetermined. In order to close the model, we are therefore required to specify constitutively this motion; this issue is considered in detail in [9].

The equations governing the multiphase mixture, interstitial flow and the nutrient transport within the microscale domain are given by (2.1)-(2.6) and (2.10)-(2.12) in Holden et al. [9]; boundary conditions are specified in (2.13)-(2.17). This microscale model is nondimensionalised via scalings detailed in $\S 2.2$ in [9]. We note that alternatives to the boundary conditions describing the cellscaffold interactions, and interfacial nutrient transport specified above may be appropriate for certain specific biological systems. These are considered in Section 4.

\section{Multiple scales analysis}

Following our previous work, we reduce the degree of nonlinearity of the microscale model to enable a more straightforward multiscale analysis by linearising the equations about a uniform steady state, across $\Omega_{T}$, as follows:

$$
\theta_{n}=\theta_{n}^{*}+\delta \theta_{n, 1}+\cdots,
$$

with corresponding expansions for the other model variables, and where $0<\delta \ll 1$ and asterisks denote steady-state values. The linearised model is given by equations (2.18)-(2.22) in [9].

We now work with the linearised version of the model and, for the sake of clarity, suppress the associated subscripts. To derive a suitable macroscale description incorporating the microscale growth, dynamics and structure, we follow (e.g.) $[5,20,24]$ in using the method of multiple scales. Correspondingly we rescale such that the timescale under consideration is that of macroscale advection and the pressure scaling results in the appropriate leading order problem:

$$
t=\varepsilon \tilde{t}, p=\frac{1}{\varepsilon} \tilde{p}
$$

in which tildes denote the rescaled variables. This choice of time rescaling results in a quasi-steady problem at leading order, thereby simplifying the analysis. Correspondingly, as in [9], we rescale growth and uptake processes to $O(\varepsilon)$. In the subsequent sections, we drop the tilde notation for convenience as we work exclusively with the rescaled variables. 
Next we introduce a macroscale coordinate $\boldsymbol{X}$ where $\boldsymbol{X}=\varepsilon \boldsymbol{x}$ ( $\boldsymbol{x}$ being the microscale coordinate) and expand all variables in the general multiple-scales form as follows:

$$
\begin{aligned}
& \psi(\boldsymbol{x}, \boldsymbol{X}, t ; \varepsilon)=\psi^{(0)}(\boldsymbol{x}, \boldsymbol{X}, t)+\varepsilon \psi^{(1)}(\boldsymbol{x}, \boldsymbol{X}, t)+\ldots \\
& \boldsymbol{\nabla}=\boldsymbol{\nabla}_{x}+\varepsilon \boldsymbol{\nabla}_{X}, \nabla^{2}=\nabla_{x}^{2}+2 \varepsilon \boldsymbol{\nabla}_{x} \cdot \boldsymbol{\nabla}_{X}+\varepsilon^{2} \nabla_{X}^{2}
\end{aligned}
$$

Moreover, in addition to the boundary conditions (2.13)-(2.17) we require that $\psi^{(i)}$ for $i=0,1, \ldots$ are periodic in $\boldsymbol{x}$. Analysing the equations at each order, and via the averaging process over the domain $\Omega_{I}$

$$
\langle g\rangle=\frac{1}{|\Omega|} \int_{\Omega_{I}} g \mathrm{~d} V,
$$

(where $\Omega=\Omega_{I} \cup \Omega_{T} \cup \Omega_{S}$ ) allows us to obtain a description of the macroscale growth and transport.

\subsection{Velocity and pressure ansatz}

Following arguments in $[6,9,20]$ we find that pressures, nutrient concentrations and cell volume fraction are independent of the microscale variable $\boldsymbol{x}$, i.e.

$$
\begin{aligned}
& p^{(0)}(\boldsymbol{X}, t)=p_{I}^{(0)}(\boldsymbol{X}, t)=p_{w}^{(0)}(\boldsymbol{X}, t)+\theta_{n}^{*} \phi_{n}^{(0)}(\boldsymbol{X}, t), \\
& c^{(0)}(\boldsymbol{X}, t)=c_{T}^{(0)}(\boldsymbol{X}, t)=c_{I}^{(0)}(\boldsymbol{X}, t), \\
& \theta_{n}^{(0)}=\theta_{n}^{(0)}(\boldsymbol{X}, t),
\end{aligned}
$$

in which $p^{(0)}$ and $c^{(0)}$ denote the overall leading-order pressure and concentration, which is uniform across $\Omega_{T} \cup \Omega_{I}$.

The governing equations at $O(\varepsilon)$ are given by (3.6)-(3.8) in [9], and therein, we followed, e.g., $[6,7,16,20,24]$ in exploiting the linearity of the $O(\varepsilon)$ momentum equations by taking an appropriate Darcy-type form for the macroscale velocities and microscale pressures to be given by the following ansatz:

$$
\boldsymbol{v}_{i}^{(0)}=-\boldsymbol{K}_{i} \boldsymbol{\nabla}_{\boldsymbol{X}} p^{(0)} \text { and } p_{i}^{(1)}=-\boldsymbol{a}_{i} \cdot \boldsymbol{\nabla}_{\boldsymbol{X}} p^{(0)}-\bar{p}_{i}, \text { for } i \in\{n, w, I\} .
$$

In (9), $\boldsymbol{K}_{i}$ are tensors describing the permeability, $\boldsymbol{a}_{i}$ are first order tensors imparting microscale pressure variation, and $\bar{p}_{i}$ are the mean (microscale-invariant) values of the first order pressures in $\Omega_{I}$.

This choice of ansatz results in unit cell problems that are parameterised by the macroscale pressure and cell volume fraction (through $\phi^{(0)}$ ) so that the micro- and macro-scale descriptions are fully-coupled (see equations (3.12)-(3.14) in [9]). This provides a significant challenge from a computational point of view. Here, we seek to remove this complexity; since both the macroscale water pressure, and pressures associated with active cell behaviour terms, appear linearly in the momentum equations $(3.6 \mathrm{c}, \mathrm{d})$ and $(3.7 \mathrm{~b})$, a more appropriate form for the macroscale velocities and microscale pressures is given by:

$$
\begin{aligned}
& \boldsymbol{v}_{i}^{(0)}=-\boldsymbol{K}_{i} \boldsymbol{\nabla}_{\boldsymbol{X}} p_{w}^{(0)}-\boldsymbol{M}_{i} \boldsymbol{\nabla}_{\boldsymbol{X}} \phi_{n}^{(0)}, \\
& p_{i}^{(1)}=-\boldsymbol{a}_{i} \cdot \boldsymbol{\nabla}_{\boldsymbol{X}} p_{w}^{(0)}-\boldsymbol{b}_{i} \cdot \boldsymbol{\nabla}_{\boldsymbol{X}} \phi_{n}^{(0)}-\bar{p}_{i},
\end{aligned}
$$

for $i \in\{n, w\}$. In the interstitial domain, the original ansatz (9) remains suitable. Note that rather than an ansatz in terms of the overall macroscale pressure $p^{(0)}$, in equations (10), (11) the macroscale velocities and microscale pressures are given as linear functions of the macroscale common mixture pressure $p_{w}^{(0)}$ and extra pressure due to cell aggregation $\phi_{n}^{(0)}$.

\subsection{Microscale cell problems}

Substituting (10), (11) into the conservation of mass and momentum equations $(3.2 \mathrm{a}, \mathrm{b})$ and $(3.6 \mathrm{c}, \mathrm{d})$ in [9] (recalling that the mass transfer terms have been rescaled to $O(\varepsilon)$ ), we obtain the following 
modified Stokes-type cell problems in $\Omega_{T}$

$$
\begin{aligned}
& \nabla_{\boldsymbol{x}} \cdot \boldsymbol{K}_{n}^{\top}=\mathbf{0}, \quad \boldsymbol{\nabla}_{\boldsymbol{x}} \cdot \boldsymbol{M}_{n}^{\top}=\mathbf{0}, \\
& \boldsymbol{\nabla}_{\boldsymbol{x}} \cdot \boldsymbol{K}_{w}^{\top}=\mathbf{0}, \quad \boldsymbol{\nabla}_{\boldsymbol{x}} \cdot \boldsymbol{M}_{w}^{\top}=\mathbf{0}, \\
& \boldsymbol{\nabla}_{\boldsymbol{x}} \boldsymbol{a}_{n}^{\top}-\boldsymbol{I}-\nabla_{\boldsymbol{x}}^{2} \boldsymbol{K}_{n}-\beta \theta_{w}^{*}\left(\boldsymbol{K}_{w}-\boldsymbol{K}_{n}\right)=\mathbf{0}, \\
& \boldsymbol{\nabla}_{\boldsymbol{x}} \boldsymbol{a}_{w}^{\top}-\boldsymbol{I}-\mu \nabla_{\boldsymbol{x}}^{2} \boldsymbol{K}_{w}-\beta \theta_{n}^{*}\left(\boldsymbol{K}_{n}-\boldsymbol{K}_{w}\right)=\mathbf{0}, \\
& \boldsymbol{\nabla}_{\boldsymbol{x}} \boldsymbol{b}_{n}^{\top}-\boldsymbol{I}-\nabla_{\boldsymbol{x}}^{2} \boldsymbol{M}_{n}-\beta \theta_{w}^{*}\left(\boldsymbol{M}_{w}-\boldsymbol{M}_{n}\right)=\mathbf{0}, \\
& \boldsymbol{\nabla}_{\boldsymbol{x}} \boldsymbol{b}_{w}^{\top}-\mu \nabla_{\boldsymbol{x}}^{2} \boldsymbol{M}_{w}-\beta \theta_{n}^{*}\left(\boldsymbol{M}_{n}-\boldsymbol{M}_{w}\right)=\mathbf{0} .
\end{aligned}
$$

In $\Omega_{I}$, standard Stokes problems are obtained via (3.3a) and (3.7b) [9], as follows:

$$
\begin{aligned}
& \nabla_{\boldsymbol{x}} \cdot \boldsymbol{K}_{I}^{\top}=\mathbf{0}, \\
& \boldsymbol{\nabla}_{\boldsymbol{x}} \boldsymbol{a}_{I}^{\top}-\boldsymbol{I}-\mu \nabla_{\boldsymbol{x}}^{2} \boldsymbol{K}_{I}=\mathbf{0} .
\end{aligned}
$$

These cell problems are coupled together through the boundary conditions $(3.4 \mathrm{a}-\mathrm{c})$ and $(3.8 \mathrm{~d})$ $[9]$ specified on the interface, $\Gamma$, which supply

$$
\begin{aligned}
& \boldsymbol{K}_{I}^{\top} \boldsymbol{n}=\mathbf{0}, \quad \boldsymbol{K}_{n}^{\top} \boldsymbol{n}=\mathbf{0}, \quad \boldsymbol{K}_{w}^{\top} \boldsymbol{n}=\mathbf{0}, \quad \boldsymbol{M}_{n}^{\top} \boldsymbol{n}=\mathbf{0}, \quad \boldsymbol{M}_{w}^{\top} \boldsymbol{n}=\mathbf{0}, \\
& -\boldsymbol{a}_{T} \otimes \boldsymbol{n}+\left(\boldsymbol{\nabla} \boldsymbol{K}_{T}+\left(\nabla \boldsymbol{K}_{T}\right)^{\top}\right) \boldsymbol{n}=-\boldsymbol{a}_{I} \otimes \boldsymbol{n}+\mu\left(\nabla \boldsymbol{K}_{I}+\left(\nabla \boldsymbol{K}_{I}\right)^{\top}\right) \boldsymbol{n}, \\
& -\boldsymbol{b}_{T} \otimes \boldsymbol{n}+\left(\boldsymbol{\nabla} \boldsymbol{M}_{T}+\left(\boldsymbol{\nabla} \boldsymbol{M}_{T}\right)^{\top}\right) \boldsymbol{n}=\theta_{n}^{*}\left[-\boldsymbol{a}_{I} \otimes \boldsymbol{n}+\mu\left(\boldsymbol{\nabla} \boldsymbol{K}_{I}+\left(\boldsymbol{\nabla} \boldsymbol{K}_{I}\right)^{\top}\right) \boldsymbol{n}\right],
\end{aligned}
$$

in which (6) has been employed to replace $p^{(0)}$, and where

$$
\begin{aligned}
& \boldsymbol{K}_{T}=\theta_{n}^{*} \boldsymbol{K}_{n}+\mu \theta_{w}^{*} \boldsymbol{K}_{w}, \quad \boldsymbol{a}_{T}=\theta_{n}^{*} \boldsymbol{a}_{n}+\theta_{w}^{*} \boldsymbol{a}_{w}, \\
& \boldsymbol{M}_{T}=\theta_{n}^{*} \boldsymbol{M}_{n}+\mu \theta_{w}^{*} \boldsymbol{M}_{w}, \quad \boldsymbol{b}_{T}=\theta_{n}^{*} \boldsymbol{b}_{n}+\theta_{w}^{*} \boldsymbol{b}_{w} .
\end{aligned}
$$

Lastly, on $\partial \Omega_{S}$, no-slip and no-penetration provides

$$
\boldsymbol{K}_{n}=\mathbf{0}, \quad \boldsymbol{K}_{w}=\mathbf{0}, \quad \boldsymbol{M}_{n}=\mathbf{0}, \quad \boldsymbol{M}_{w}=\mathbf{0} .
$$

For uniqueness in the above cell problems, we use a standard approach (see, e.g. $[17,20,23,24])$ and impose that in the relevant domain

$$
\left\langle\boldsymbol{a}_{i}\right\rangle=\mathbf{0}, \quad\left\langle\boldsymbol{b}_{i}\right\rangle=\mathbf{0} .
$$

We note that, while a standard Stokes-type cell problem is obtained in $\Omega_{I}$, the multiphase dynamics in $\Omega_{T}$ leads to significantly increased complexity. In particular, we obtain a set of coupled modified Stokes problems, determining the permeability tensors $\boldsymbol{K}_{i}, \boldsymbol{M}_{i}$ and extra pressures $\boldsymbol{a}_{i}, \boldsymbol{b}_{i}$ for each phase, which are further coupled to the flow in $\Omega_{I}$ via stress and velocity continuity boundary conditions. Furthermore, we highlight that whilst the number of cell problems has increased as a result of the change in ansatz from that employed in [9], we find that the permeability tensors are no longer dependent on macroscale pressures. The system we obtain therefore represents a significant simplification, taking the more familiar form, where the quasi-steady cell problems can be solved separately from the macroscale description, that we obtain below.

\subsection{Averaging}

The macroscale flow is obtained by averaging (11) via the definition (5) to obtain

$$
\left\langle\boldsymbol{v}_{i}^{(0)}\right\rangle=-\left\langle\boldsymbol{K}_{i}\right\rangle \boldsymbol{\nabla}_{\boldsymbol{X}} p_{w}^{(0)}-\left\langle\boldsymbol{M}_{i}\right\rangle \boldsymbol{\nabla}_{\boldsymbol{X}} \phi_{n}^{(0)},
$$


wherein $p_{w}^{(0)}$ and $\phi_{n}^{(0)}$ (via $\theta_{n}^{(0)}$, and equations (2.6), (2.19) in [9]) are obtained from the following system, derived from the average (exploiting the divergence theorem) of equations $(3.6 \mathrm{a}, \mathrm{b}, \mathrm{e})$ and $(3.7 \mathrm{a}, \mathrm{c})$ :

$$
\begin{aligned}
& \frac{\partial}{\partial t}\left\langle\theta_{n}^{(0)}\right\rangle_{T}+\theta_{n}^{*}\left(\boldsymbol{\nabla}_{\boldsymbol{X}} \cdot\left\langle\boldsymbol{v}_{n}^{(0)}\right\rangle_{T}+\left\langle\boldsymbol{v}_{\Gamma}^{(1)} \cdot \boldsymbol{n}\right\rangle_{\Gamma}\right)=\left\langle S_{n}^{(0)}\right\rangle_{T}, \\
& \boldsymbol{\nabla}_{\boldsymbol{X}} \cdot\left(\tilde{\boldsymbol{K}} \boldsymbol{\nabla}_{\boldsymbol{X}} p_{w}^{(0)}+\tilde{\boldsymbol{M}} \boldsymbol{\nabla}_{\boldsymbol{X}} \phi_{n}^{(0)}\right)=-\left\langle(1-\bar{\rho}) S_{n}^{(0)}\right\rangle_{T}, \\
& \Phi_{T \cup I} \frac{\partial c^{(0)}}{\partial t}+c^{*}(1-\bar{\rho})\left\langle S_{n}^{(0)}\right\rangle_{T}=-\left\langle\Lambda^{(0)}\right\rangle_{T},
\end{aligned}
$$

where $\Phi_{T \cup I}=\left|\Omega_{T} \cup \Omega_{I}\right| /|\Omega|$. We note that equation (30) arises from the average of the sum of (3.6e) and (3.7c) in [9]; on application of the divergence theorem and boundary conditions, terms associated with $c_{T}^{(1)}$ and $c_{I}^{(1)}$ vanish.

The tensors $\tilde{\boldsymbol{K}}$ and $\tilde{\boldsymbol{M}}$ are given by

$$
\tilde{\boldsymbol{K}}=\left\langle\theta_{n}^{*} \boldsymbol{K}_{n}+\theta_{w}^{*} \boldsymbol{K}_{w}\right\rangle_{T}+\left\langle\boldsymbol{K}_{I}\right\rangle_{I}, \quad \tilde{\boldsymbol{M}}=\left\langle\theta_{n}^{*} \boldsymbol{M}_{n}+\theta_{w}^{*} \boldsymbol{M}_{w}\right\rangle_{T}+\left\langle\theta_{n}^{*} \boldsymbol{K}_{I}\right\rangle_{I},
$$

where the individual permeability tensors $\boldsymbol{K}_{i}$ and $\boldsymbol{M}_{i}$ are determined from the set of coupled Stokes problems (12)-(22), (25), (26).

We remark that while the modification to the unit cell problems outlined above is significant, the impact of our modification to the approach of [9] on the macroscale description is less significant, being restricted to the redefinition of the relevant permeability tensors, and the associated velocities and pressures (in particular in the the explicit appearance of $\boldsymbol{\nabla}_{\boldsymbol{X}} \phi_{n}^{(0)}$ terms associated with active cell motion). The governing system itself is of identical structure, and comprises a macroscale Darcy flow PDE, coupled to reaction equations describing tissue component volume fractions and nutrient concentration. Lastly, we note that as is common in analyses of this type, the macroscale model we obtain is not closed: we are required to specify constitutively the $\mathrm{O}(\varepsilon)$ boundary velocity $\boldsymbol{v}_{\Gamma}^{(1)} \cdot \boldsymbol{n}$ $(c f .[6,10])$. This is explored in [9] by means of detailed investigation of the travelling wave properties of the microscale multiphase model, but we do not pursue this here.

Lastly, we note in passing that in the limit case of inviscid water (that was employed in [9] for illustrative numerical simulations), the overall pressure $p^{(0)}$ is zero and consequently so is $p_{w}^{(0)}+$ $\theta_{n}^{*} \phi_{n}^{(0)}$. This means that the new ansatz $(10),(11)$ can be rewritten as

$$
\begin{aligned}
& \boldsymbol{v}_{i}^{(0)}=\left[-\boldsymbol{K}_{i}+\frac{1}{\theta_{n}^{*}} \boldsymbol{M}_{i}\right] \boldsymbol{\nabla}_{\boldsymbol{X}} p_{w}^{(0)}, \\
& p_{i}^{(1)}=\left[-\boldsymbol{a}_{i}+\frac{1}{\theta_{n}^{*}} \boldsymbol{b}_{i}\right] \boldsymbol{\nabla}_{\boldsymbol{X}} p_{w}^{(0)}-\bar{p}_{i},
\end{aligned}
$$

which is equivalent to (9), where the terms in square brackets are given by single tensors.

\section{Alternative boundary conditions}

In the model described above, we impose no-slip and no-penetration conditions on the scaffold boundary $\Omega_{S}$ and continuity of nutrient flux and concentration on the free interface $\Gamma$. While these are physically sensible choices in general, reflecting the solid nature of the scaffold material and passive diffusive transport of nutrient into the tissue domain, in some cases, a less restrictive choice may be of interest. For example, as well as the active motion embodied by the intraphase pressure $\phi_{n}$, cells may exhibit significant haptotactic motion on the scaffold surface itself. This is especially pertinent to the tissue engineering application under study, in which scaffolds may be manufactured to incorporate substrate-bound chemoattractants thereby promoting cell ingress (see, e.g., $[18,19,26]$ and references therein). Additionally, other descriptions for solute transport across $\Gamma$ may be appropriate, for example accommodating aspects of active transport, binding kinetics or membrane law behaviour. 


\subsection{Cell motion on the scaffold surface}

As a simple alternative choice of boundary condition permitting cell motion on the scaffold surface, we now consider a slip condition of the form

$$
\boldsymbol{v}_{n}=b \frac{\partial \boldsymbol{v}_{n}}{\partial n}, \boldsymbol{x} \in \Gamma
$$

where $b$ is a constant of proportionality and $\partial / \partial n$ denotes the normal derivative. We retain the no-penetration condition $\boldsymbol{v}_{i} \cdot \boldsymbol{n}=0$ on $\partial \Omega_{S}$ since the scaffold is assumed to remain solid. We remark, however, that it would be straightforward to accommodate, for example, scaffold dissolution, invoking the approach and scaling arguments in [20].

The effective macroscale equations remain the same in each case and the only change to the Stokes problem is in the tissue-scaffold boundary conditions. The above equations give

$$
\boldsymbol{K}_{n}=b \frac{\partial \boldsymbol{K}_{n}}{\partial n}, \quad \boldsymbol{M}_{n}=b \frac{\partial \boldsymbol{M}_{n}}{\partial n}, \quad \boldsymbol{K}_{n}^{\top} \boldsymbol{n}=\mathbf{0}, \quad \boldsymbol{M}_{n}^{\top} \boldsymbol{n}=\mathbf{0}
$$

as the set of alternative boundary conditions to be applied on $\boldsymbol{K}_{n}$ and $\boldsymbol{M}_{n}$ at $\partial \Omega_{S}$ in the Stokes problem. (Note that the slip condition is of similar form to that obtained by Irons et al. [10] in a similar cell problem, for a porous medium growth model.)

\subsection{Nutrient flux}

As noted above, we have imposed continuity of flux and concentration on the tissue-interstitium interface $\Gamma$. For completeness, we also indicate the influence of alternative concentration flux boundary conditions. As discussed in [24] two further, widely-used, options are suggested, which we now consider:

Option 1 - Membrane law The flux of nutrient concentration across the boundary is proportional to the concentration jump. This widely-used approach demands:

$$
\left(c_{I} \boldsymbol{v}_{I}-D_{I} \boldsymbol{\nabla} c_{I}\right) \cdot \boldsymbol{n}=\left(c_{T} \boldsymbol{v}_{T}-D_{T} \boldsymbol{\nabla} c_{T}\right) \cdot \boldsymbol{n}=r\left(c_{T}-c_{I}\right),
$$

where $r$ is a constant reflecting the permeability of the tissue boundary to nutrient flux.

Option 2 - Concentration jump due to species solubility Alternatively, a concentration jump may be permitted, as a consequence of reduced solvability in the tissue compared to the interstitium ( $c f$. Henry's law for gases in which the concentration $c$ and partial pressure $P$ of a gas in solution are related through the $c=\gamma P$, where $\gamma$ denotes the solvability):

$$
\alpha c_{I}=c_{T},
$$

where we assume for simplicity that $\alpha$ is a constant, although in a more general formulation it may be suitable to specify $\alpha=\alpha\left(\theta_{n}\right)$.

In the following, we investigate the choice of boundary condition, and scaling of associated constant, on the effective macroscale description. Note that the choice of condition has no direct impact on the Stokes problem on the periodic cell.

\subsubsection{Membrane law}

Firstly we linearise the boundary condition (35); assuming that at steady state the nutrient concentration is equal and uniform across both domains, $\Omega_{I}$ and $\Omega_{T}$ we obtain:

$$
c^{*}\left(\boldsymbol{v}_{I}-\boldsymbol{v}_{\Gamma}-\frac{1}{P e_{I}} \boldsymbol{\nabla} c_{I}\right) \cdot \boldsymbol{n}=c^{*}\left(\boldsymbol{v}_{T}-\boldsymbol{v}_{\Gamma}-\frac{1}{P e_{T}} \boldsymbol{\nabla} c_{T}\right) \cdot \boldsymbol{n}=r\left(c_{T}-c_{I}\right) .
$$


In the subsequent multiple scales analysis we consider two further scaling subcases on the membrane permeability; namely $r=\mathrm{O}(1)$ or $r=\varepsilon \bar{r}$, with $\bar{r}=\mathrm{O}(1)$. At leading order the boundary condition reads in each case:

$$
-\frac{1}{P e_{I}} \nabla_{\boldsymbol{x}} c_{I}^{(0)} \cdot \boldsymbol{n}=-\frac{1}{P e_{T}} \nabla_{\boldsymbol{x}} c_{T}^{(0)} \cdot \boldsymbol{n}=\left\{\begin{array}{l}
r\left(c_{T}^{(0)}-c_{I}^{(0)}\right) \\
0
\end{array}\right.
$$

We recall that the leading order problem is quasi-steady, so there is no growth of $\Omega_{T}$, flux of fluid across the interface or nutrient uptake; correspondingly, and in line with the linearised model set-up, it is consistent to assume that there is no induced diffusive transport of nutrient across $\Gamma$ either. In the first sub-case $(r=O(1))$ this implies that, since $r \neq 0, c_{T}^{(0)}=c_{I}^{(0)}$, whilst in the second sub-case $(r=\varepsilon \bar{r})$ there is already no interfacial transport at this order and a concentration jump can arise. In both cases, the leading order concentration in each domain is independent of $\boldsymbol{x}$.

Following through the rest of the analysis as described above and in [9] for $\mathrm{O}(1)$ membrane permeability, we find that the effective macroscale equation is unchanged,

$$
\Phi_{T \cup I} \frac{\partial c^{(0)}}{\partial t}+c^{*}(1-\bar{\rho})\left\langle S_{n}^{(0)}\right\rangle_{T}=-\left\langle\Lambda^{(0)}\right\rangle_{T} .
$$

In the second sub-case, there are minor differences imbued by the fact that a leading-order concentration jump may be permitted and we obtain the following macroscale representation in each domain

$$
\begin{aligned}
\Phi_{T} \frac{\partial c_{T}^{(0)}}{\partial t}+c^{*}(1-\bar{\rho})\left\langle S_{n}^{(0)}\right\rangle_{T} & =-\left\langle\bar{r}\left(c_{T}^{(0)}-c_{I}^{(0)}\right)\right\rangle_{\Gamma}-\left\langle\Lambda^{(0)}\right\rangle_{T} \\
\Phi_{I} \frac{\partial c_{I}^{(0)}}{\partial t} & =\left\langle\bar{r}\left(c_{T}^{(0)}-c_{I}^{(0)}\right)\right\rangle_{\Gamma},
\end{aligned}
$$

which are identical to those presented in [24], except that advective transport is linearised in our description. Macroscale nutrient concentration in this case is given by two coupled equations, one for each of $c_{T}^{(0)}$ and $c_{I}^{(0)}$.

\subsubsection{Concentration jump due to species solubility}

We remark that when linearising the model equations we can no longer assume that at steady state nutrient concentration $c^{*}$ is uniform across the entire unit cell (unless $\alpha=1$, which returns us our original representation). We instead suppose that nutrient concentration is uniform in each domain, connected by the boundary condition, i.e.

$$
\begin{aligned}
c_{T} & =c_{T}^{*}+\delta c_{T 1}+\ldots \\
c_{I} & =c_{I}^{*}+\delta c_{I 1}+\ldots
\end{aligned}
$$

where

$$
\alpha c_{I}^{*}=c_{T}^{*}
$$

and the steady state concentrations $c_{T}^{*}$ are defined as in $\S 2.3$ in [9].

The linearised and rescaled equations for the nutrient concentration are given by:

$$
\begin{aligned}
& \varepsilon \frac{\partial c_{T, 1}}{\partial t}+\nabla \cdot\left(c_{T}^{*}\left(\theta_{n}^{*} \boldsymbol{v}_{n, 1}+\theta_{w}^{*} \boldsymbol{v}_{w, 1}\right)\right)=\frac{1}{P e_{T}} \nabla^{2} c_{T, 1}-\Lambda_{1} \text { in } \Omega_{T}, \\
& \varepsilon \frac{\partial c_{I, 1}}{\partial t}+\nabla \cdot\left(c_{I}^{*} \boldsymbol{v}_{I, 1}\right)=\frac{1}{P e_{I}} \nabla^{2} c_{I, 1} \text { in } \Omega_{I}, \\
& {\left[c_{i}^{*}\left(\boldsymbol{v}_{i, 1}-\boldsymbol{v}_{\Gamma, 1}\right) \cdot \boldsymbol{n}-\frac{1}{P e_{i}} \nabla c_{i, 1} \cdot \boldsymbol{n}\right]_{-}^{+}=0,} \\
& \alpha c_{I, 1}=c_{T, 1} \text { on } \Gamma .
\end{aligned}
$$


All other equations remain unchanged from the original analysis, except that in the cell proliferation and nutrient uptake terms $c^{*}$ is replaced by $c_{T}^{*}$. In the following, the subscripts associated with the linearisation are omitted for clarity.

At leading order, we find, via standard arguments, that both $c_{I}^{(0)}$ and $c_{T}^{(0)}$ are independent of the microscale and related by the leading order version of (48).

At $\mathrm{O}(\varepsilon)$ the relevant equations are:

$$
\begin{aligned}
& \frac{\partial c_{T}^{(0)}}{\partial t}+c_{T}^{*}\left(\boldsymbol{\nabla}_{\boldsymbol{x}} \cdot\left(\theta_{n}^{*} \boldsymbol{v}_{n}^{(1)}+\theta_{w}^{*} \boldsymbol{v}_{w}^{(1)}\right)+\boldsymbol{\nabla}_{\boldsymbol{X}} \cdot\left(\theta_{n}^{*} \boldsymbol{v}_{n}^{(0)}+\theta_{w}^{*} \boldsymbol{v}_{w}^{(0)}\right)\right) \\
& =\frac{1}{P e_{T}} \nabla_{\boldsymbol{x}}^{2} c_{T}^{(1)}-\Lambda^{(0)} \text { in } \Omega_{T}, \\
& \frac{\partial c_{I}^{(0)}}{\partial t}+c_{I}^{*}\left(\boldsymbol{\nabla}_{\boldsymbol{x}} \cdot \boldsymbol{v}_{I}^{(1)}+\boldsymbol{\nabla}_{\boldsymbol{X}} \cdot \boldsymbol{v}_{I}^{(0)}\right)=\frac{1}{P e_{I}} \boldsymbol{\nabla}_{\boldsymbol{x}}^{2} c_{I}^{(1)} \text { in } \Omega_{I}, \\
& {\left[c_{i}^{*}\left(\boldsymbol{v}_{i}^{(1)}-\boldsymbol{v}_{\Gamma}^{(1)}\right) \cdot \boldsymbol{n}-\frac{1}{P e_{i}}\left(\boldsymbol{\nabla}_{\boldsymbol{x}} c_{i}^{(1)}+\boldsymbol{\nabla}_{\boldsymbol{X}} c_{i}^{(0)}\right) \cdot \boldsymbol{n}\right]_{-}^{+}=0,} \\
& \alpha c_{I}^{(1)}=c_{T}^{(1)} \text { on } \Gamma .
\end{aligned}
$$

On averaging (49) and (50) over their domains, we obtain:

$$
\begin{aligned}
\Phi_{T} \frac{\partial c_{T}^{(0)}}{\partial t}+c_{T}^{*}(1-\bar{\rho})\left\langle S_{n}^{(0)}\right\rangle_{T} & =\left\langle\frac{1}{P e_{T}} \nabla_{\boldsymbol{x}} c_{T}^{(1)} \cdot \boldsymbol{n}\right\rangle_{\Gamma}-\left\langle\Lambda^{(0)}\right\rangle_{T} \\
\Phi_{I} \frac{\partial c_{I}^{(0)}}{\partial t} & =-\left\langle\frac{1}{P e_{I}} \nabla_{\boldsymbol{x}} c_{I}^{(1)} \cdot \boldsymbol{n}\right\rangle_{\Gamma} .
\end{aligned}
$$

Averaging boundary condition (51) over $\Gamma$ and rearranging, we find that

$$
\left\langle\frac{1}{P e_{T}} \nabla_{\boldsymbol{x}} c_{T}^{(1)} \cdot \boldsymbol{n}\right\rangle_{\Gamma}-\left\langle\frac{1}{P e_{I}} \boldsymbol{\nabla}_{\boldsymbol{x}} c_{I}^{(1)} \cdot \boldsymbol{n}\right\rangle_{\Gamma}=\left(c_{T}^{*}-c_{I}^{*}\right)\left\langle Q^{(0)}\right\rangle_{\Gamma},
$$

where

$$
Q^{(0)}=\left(\boldsymbol{v}_{I}^{(1)}-\boldsymbol{v}_{\Gamma}^{(1)}\right) \cdot \boldsymbol{n}=\left(\boldsymbol{v}_{T}^{(1)}-\boldsymbol{v}_{\Gamma}^{(1)}\right) \cdot \boldsymbol{n}=\theta_{w}^{*}\left(\boldsymbol{v}_{w}^{(1)}-\boldsymbol{v}_{\Gamma}^{(1)}\right) \cdot \boldsymbol{n}
$$

describes the leading order flux of material across the boundary of the tissue domain.

Summing (53) and (54), and exploiting (44) and (48) to eliminate $c_{I}^{*}$ and $c_{I}^{(0)}$, we obtain

$$
\left(\Phi_{T}+\frac{1}{\alpha} \Phi_{I}\right) \frac{\partial c_{T}^{(0)}}{\partial t}+c_{T}^{*}(1-\bar{\rho})\left\langle S_{n}^{(0)}\right\rangle_{T}=c_{T}^{*}\left(1-\frac{1}{\alpha}\right)\left\langle Q^{(0)}\right\rangle_{\Gamma}-\left\langle\Lambda^{(0)}\right\rangle_{T},
$$

and $Q^{(0)}$ must be determined. Note that when $\alpha=1$, i.e. we have continuity of concentration on the boundary, we obtain (30) as in the original model, and $Q^{(0)}$ no longer appears.

\section{Discussion}

In this paper, we have revisited and extended the work of [9] to derive a new effective description for a growing tissue, by means of two-scale asymptotics. We considered a rigid periodic latticelike structure covered by a layer of growing tissue. The model is therefore applicable to problems in regenerative medicine, such as tissue growth within a tissue engineering scaffold (our primary motivation), or biofilm growth, for example in the subsurface or the fouling of filters.

Multiscale homogenisation techniques are increasing in popularity in biologically-inspired models, with a recent series of studies seeking to incorporate growth $[4,6,9,20,23]$. As in [9], here, we seek to accommodate a more complex description of tissue growth than one comprising a solid undergoing accretion [20,23] or volumetric growth [4], by employing a multiphase fluid tissue model that naturally accommodates aspects such as interstitial growth and active cell motion, while still 
obtaining a tractable macroscale description. (A multiphase approach was used in [6]; however, exploiting the limit of large interphase drag reduces the dynamics to effectively an accretion-type process.) In [9], this deficiency was addressed to obtain an effective description of tissue growth that retains active cell motion, caused by cellular aggregation or repulsion. Analytical progress was effected by a linearisation that ameliorates problems associated with complex mass-transfer considered in the multiphase model; however, the macroscale description obtained was fully coupled to the microscale unit cell problems, thereby providing a significant computational challenge in the general case (decoupling is obtained in the inviscid limit case). Here, we address this feature by adopting a more suitable solution ansatz to describe the velocities and pressures in the system, that respects the linear structure of the relevant momentum equations. This analysis provides a macroscale model of very similar structure to that presented in [9], parameterised by permeability tensors, provided by a set of modified Stokes-type cell problems. The contribution of this work is that, unlike that presented in [9], the cell problems are independent of the macroscale description, leading to a system whereby the quasi-steady cell problems may be solved separately from the macroscale description, thereby greatly simplifying the computational difficulty associated with fully-coupled multiscale descriptions. Moreover, we also demonstrate how the model formulation is changed under a set of exemplar alternative microscale boundary conditions associated with, for example, cell motion over the scaffold surface, or alternative nutrient flux dynamics across the tissue-interstitium boundary.

\section{References}

[1] T. Alarcón, M.R. Owen, H.M. Byrne, and P.K. Maini. Multiscale modelling of tumour growth and therapy: the influence of vessel normalisation on chemotherapy. Comput. Math. Methods Med., 7(2-3):85-119, 2006.

[2] A. Bensoussan, J-L Lions, and G. Papanicolaou. Asymptotic analysis for periodic structures, volume 5. North-Holland Publishing Company Amsterdam, 1978.

[3] R.M. Bowen. Incompressible porous media models by use of the theory of mixtures. International Journal of Engineering Science, 18(9):1129-1148, 1980.

[4] J. Collis, D.L. Brown, M.E. Hubbard, and R.D. O'Dea. Effective equations governing an active poroelastic medium. Proc. R. Soc. A, 473(2198):20160755, 2017.

[5] J. Collis, M.E. Hubbard, and R.D. O'Dea. Computational modelling of multiscale, multiphase fluid mixtures with application to tumour growth. Comp. Meth. Appl. Mech. Eng., 309:554-578, 2016.

[6] J. Collis, M.E. Hubbard, and R.D. O'Dea. A multi-scale analysis of drug transport and response for a multi-phase tumour model. Eur. J. Appl. Math., pages 1-36, 2016.

[7] Y. Davit, C. G. Bell, H.M. Byrne, L. AC Chapman, L. S. Kimpton, G. E. Lang, K. HL Leonard, J. M. Oliver, N.C. Pearson, R.J. Shipley, et al. Homogenization via formal multiscale asymptotics and volume averaging: How do the two techniques compare? Advances in Water Resources, 62:178-206, 2013.

[8] D.A. Drew and S.L. Passman. Theory of multicomponent fluids, volume 135. Springer Science \& Business Media, 2006.

[9] E.C. Holden, J. Collis, B.S. Brook, and R.D. O'Dea. A multiphase multiscale model for nutrient limited tissue growth. ANZIAM Journal, 2018.

[10] L. Irons, J. Collis, and R.D. O'Dea. Microstructural influences on growth and transport in biological tissue - a multiscale description. In Modeling of microscale transport in biological processes, pages 311-334. Elsevier, 2017. 
[11] A.E. Jakus, E.B. Secor, A.L. Rutz, Sumanas W. Jordan, M.C. Hersam, and R.N. Shah. Threedimensional printing of high-content graphene scaffolds for electronic and biomedical applications. ACS Nano, 9(4):4636-4648, 2015.

[12] J.B. Keller. Darcy's law for flow in porous media and the two-space method. Nonlinear Partial Differential Equations in Engineering and Applied Sciences, 31:429-443, 1980.

[13] G. Lemon, J.R. King, H.M. Byrne, O.E. Jensen, and K.M. Shakesheff. Mathematical modelling of engineered tissue growth using a multiphase porous flow mixture theory. Journal of Mathematical Biology, 52(5):571-594, 2006.

[14] P. Macklin, S. McDougall, A.R.A Anderson, M.A.J. Chaplain, V. Cristini, and J. Lowengrub. Multiscale modelling and nonlinear simulation of vascular tumour growth. J. Math. Biol., 58(4-5):765-798, 2009.

[15] C.M. Marle. On macroscopic equations governing multiphase flow with diffusion and chemical reactions in porous media. International Journal of Engineering Science, 20(5):643-662, 1982.

[16] C.C. Mei and J.L. Auriault. The effect of weak inertia on flow through a porous medium. J. Fluid Mech., 222(1):647-663, 1991.

[17] C.C. Mei and B. Vernescu. Homogenization methods for multiscale mechanics. World scientific, 2010.

[18] E.D. Miller, G.W Fisher, L.E. Weiss, L.M. Walker, and P.G Campbell. Dose-dependent cell growth in response to concentration modulated patterns of fgf-2 printed on fibrin. Biomaterials, $27(10): 2213-2221,2006$.

[19] E.D. Miller, K. Li, T. Kanade, L.E. Weiss, L.M. Walker, and P.G. Campbell. Spatially directed guidance of stem cell population migration by immobilized patterns of growth factors. Biomaterials, 32(11):2775-2785, 2011.

[20] R.D O'Dea, M.R. Nelson, A.J. El Haj, S.L. Waters, and H.M. Byrne. A multiscale analysis of nutrient transport and biological tissue growth in vitro. Math. Med. Biol., 32(3):345-366, 2015.

[21] J.M. Osborne, A. Walter, S.K. Kershaw, G.R. Mirams, A.G. Fletcher, P. Pathmanathan, D. Gavaghan, O.E. Jensen, P.K. Maini, and H.M. Byrne. A hybrid approach to multi-scale modelling of cancer. Phil. Trans. R. Soc. A, 368(1930):5013-5028, 2010.

[22] G.A. Pavliotis and A. Stuart. Multiscale methods: averaging and homogenization. Springer Science \& Business Media, 2008.

[23] R. Penta, D. Ambrosi, and R.J. Shipley. Effective governing equations for poroelastic growing media. Q. J. Mech. Appl. Math., 67(1):69-91, 2014.

[24] R.J. Shipley and S.J. Chapman. Multiscale modelling of fluid and drug transport in vascular tumours. Bull. Math. Biol., 72(6):1464-1491, 2010.

[25] J. Visser, F.P.W. Melchels, J.E. Jeon, E.M. van Bussel, L.S. Kimpton, H.M. Byrne, W.J.A. Dhert, P.D. Dalton, D.W. Hutmacher, and J. Malda. Reinforcement of hydrogels using threedimensionally printed microfibres. Nature communications, 6, 2015.

[26] J.H. Wen, O. Choi, H. Taylor-Weiner, A. Fuhrmann, J.V. Karpiak, A. Almutairi, and A.J. Engler. Haptotaxis is cell type specific and limited by substrate adhesiveness. Cellular and molecular bioengineering, 8(4):530-542, 2015. 